\title{
Current Medication
}

National Cancer Institute

\section{Source}

National Cancer Institute. Current Medication. NCI Thesaurus. Code C156818.

The current medications an individual is taking. 